\title{
Propulsion system based on compressed air due to rotor blade rotation
}

\author{
Aiman Elmahmodi ${ }^{\mathrm{a}}$, Nikola Davidovic ${ }^{\mathrm{b}}$, Ramadan A. Al-Madani ${ }^{\mathrm{c}}$ \\ ${ }^{a}$ Aeronautical Eng., Faculty of engineering-University of Tripoli, Tripoli - Libya \\ ${ }^{b}$ EDePro Jet Propulsion Laboratory, Begrade, Serbia \\ ${ }^{c}$ Engineering Faculty, Al-Jabel Algharbi University, Tripoli - Libya
}

\begin{abstract}
The theoretical and experimental investigation of the potential of jet propulsion system to drive rotor of (VTOL) Vertical Takeoff and Landing aircraft has been presented. The analysis of tip jet propulsion system is based on compressed air due to rotor blade rotation. The system performances were compared in the case of the rotor with rocket, and pressure-jet engine. Rocket engine had practical application in middle of 20th century, while the present work tip jet pressure propulsion system is based on turbojet thermodynamic cycle. Comparison has been performed according to available and required power, and to flight duration. Performances of the present propulsion system were also analyzed with introduction of typical rotor geometry. Analysis shows possible application of such propulsion as unmanned or low-weight helicopters and Vertical Take-Off and Landing (VTOL) vehicles.
\end{abstract}

Keywords: Helicopter rotor, tip jet, flying vehicle, unmanned helicopters, pressure jet.

\begin{tabular}{llll}
\multicolumn{2}{l}{ Nomenclature } & & \\
$\mathrm{m}_{\mathrm{a}}$ & Air inlet mass flow rate $(\mathrm{kg} / \mathrm{sec})$ & $n$ & number of rotor blades \\
$m_{f}$ & Fuel mass flow rate $(\mathrm{kg} / \mathrm{sec})$ & $M$ & payload $(\mathrm{Kg})$ \\
$m_{e}$ & exit mass flow rate $(\mathrm{kg} / \mathrm{sec})$ & $f$ & fuel - air mixture ratio \\
$c_{p a}$ & Specific heat $(\mathrm{J} / \mathrm{kg} . \mathrm{K})$ & $P$ & Power $(\mathrm{w})$ \\
$T a$ & Ambient temperature $(\mathrm{K})$ & $P_{R O T}$ & Rotor useful power $(\mathrm{w})$ \\
$C_{s p}$ & specific fuel consumption $(\mathrm{kg} / \mathrm{Nh})$ & $P_{d r a g}$ & drag power $(\mathrm{w})$ \\
$\gamma$ & Specific heat ratio & $F$ & Thrust $(\mathrm{N})$ \\
$\pi_{r}$ & pressure ratio of the rotor blade. & $V_{e}$ & Nozzle exit Velocity $(\mathrm{m} / \mathrm{sec})$ \\
$\eta_{r}$ & efficiency of the rotor & $V_{t}$ & Rotor blade tip velocity $(\mathrm{m} / \mathrm{sec})$ \\
$\tau$ & cursing time & $W_{p}$ & Rotor compression work $(\mathrm{J} / \mathrm{kg})$.
\end{tabular}

\section{Introduction}

Tip jet driven helicopter uses the power of gases discharged through the tip of the blades of the rotor to impart rotating motion to the rotor. No torque is transferred from the fuselage to the rotor. Therefore the tail rotor may not be needed and driven system has a considerably simpler construction. Indeed, the tail rotor is a major cause of accidents and it adds complexity to the helicopter drive system. As a consequence helicopter is lighter. At the same time, some disadvantages arise from the utilization of tip jet. One major shortcoming is the low efficiency of its drive system. However, the lightened weight compensates this deficiency.

A tilt-rotor compound VTOL aircraft has a multiple-flow thrust generator(s) comprising a gaspowered tip-jet driven rotor having a thrust-augmentation ratio of at least two; that tilts about the aircraft's

\footnotetext{
* Manuscript received June 29, 2013; revised October 5, 2013.

Corresponding author. Tel.: 00218912189321; E-mail address: elmahmodi@ aerodept.edu.ly
} 
pitch axis wherein the rotor's plane of rotation is substantially horizontal for VTOL operations and the rotor's plane of rotation is substantially vertical forward flight operations. A relatively small fixed-wing sustains the aircraft during forward flight. Compressed exhaust gas from the fan-jet engines is ducted to a manifold having valves, which control power to the multiple-flow thrust generators and to the jet exhaust nozzle(s) as supplemental thrust for forward propulsion and yaw control. The manifold also serves to distribute compressed gas to the dead engine side of the aircraft in the event of a dead engine emergency, and to reaction jets for attitude control during VTOL operations. Thus, this tilt-rotor aircraft is more efficient, faster, immensely lighter, less complex and less expensive to purchase and maintain than its shaft-driven counterparts. A quick glimpse of the first test flights of Doblhoff's jet tip powered Helicopter, constructed at the Wiener Neustadter Flugzeugwerke. It was the world's first helicopter to employ jet propulsion [1]. The WNF-342 V2 shown in Fig. 1, flew successfully in 1943. It weighed approx $450 \mathrm{~kg}$. By sending out jets of compressed air from the rotor tips to spin the rotors, there is no torque and thus no need for a second anti torque rotor.

For the past several years, Georgia Tech, working closely with NASA Ames' System Analysis Branch, has been pursuing the development of a design methodology which will enable the designer to size and predict routinely the performance of stopped rotor/wing vehicles. Despite of the fact that the HSRC program is no longer funded, Georgia Tech has been pursuing the development and implementation of this methodology in an effort to understand the physics of this complicated problem and advance the state-of-the-art. The step by step description of this overall design methodology can be found in Reference [4].

The pressure jet helicopters system, also described in [5], in terms of technical concepts characteristics, emphasizing on propulsion and yaw control systems, which point out the differences between the pressure jet and conventional helicopters.
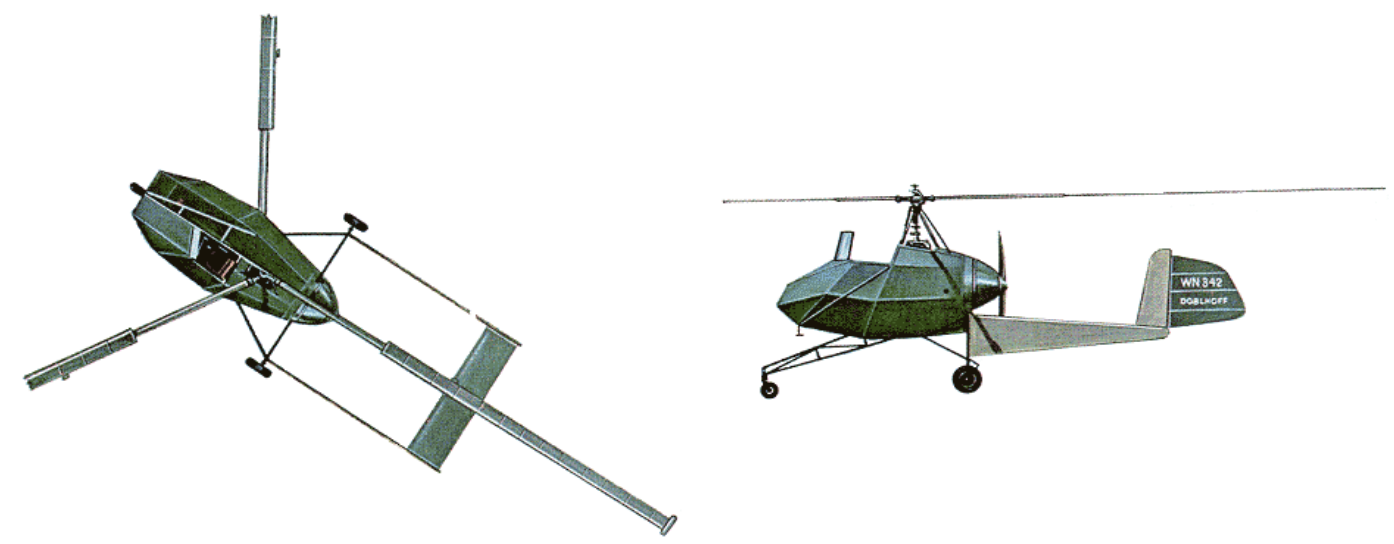

Fig. 1. Doblhoff WNF-342.

\section{Rocket Motor Tip-jet Drive}

In the rocket motor used for to the helicopter rotor tip jet, the liquid hydrogen peroxide is fed with high pressure from a tank to a chamber filled with a decomposition catalyst.

- The decomposition catalyst causes the hydrogen peroxide to decompose into water steam and oxygen. Heat is released by the reaction. The temperature of the formed gas mixture is about $650{ }^{\circ} \mathrm{C}$.

- After passing through the catalyst chamber the hot, high pressure gas mixture is released through a rocket nozzle.

- The velocity of the gas flow after the nozzle becomes well over $1000 \mathrm{~m} / \mathrm{s}$ and gives the rocket a considerable reaction force thrust.

Fig. 2 had shown rocket motor over hydrogen-peroxide that operates helicopter Intora Firebird IF. Thrust generated in the rotor tip due to rocket motoer can be presented as [6]. 


$$
F=m_{e} V_{e}
$$

Thrust power that will be generated by the rotor with two rocket motors in the tip is obtained by:

$$
P=2 F V_{t}=2 m_{e} V_{e} V_{t}
$$

Specific power and, specific fuel consumption for the two rocket motors are obtained by:

$$
\begin{aligned}
& P_{s p}=\frac{N}{2 m_{e}}=V_{e} V_{t} \\
& C_{s p}=\frac{2 m_{e}}{P}=\frac{1}{P_{s p}}=\frac{1}{V_{e} V_{t}}
\end{aligned}
$$

In the above-mentioned equations $\mathrm{m}_{\mathrm{e}}$ masseurs combustion chamber hot gases mass flow, $\mathrm{V}_{\mathrm{e}}$ nozzle outlet velocity, and $\mathrm{V}_{\mathrm{t}}$ is rotor blade tip speed.

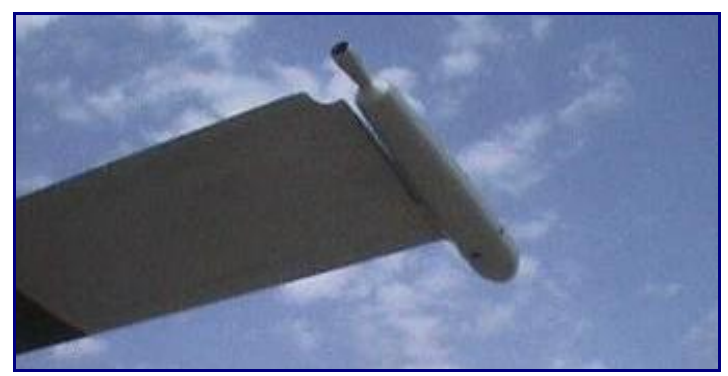

Fig. 2. Rocket motor ashore rotor helicopter Intora Firebird.

\section{Present Developed Pressure Power System}

In the pressure combustion system, air is compressed through the blade rotor due to centrifugal force generated during blade rotation. What characterizing this method is that, no air compressor unit is used. Incoming air is compressed in the rotor and directed to 2-D combustion chamber located at the end of the rotor, and then ejected through the nozzle to produce thrust force. This thermo-dynamics circle is identical to that of turbojet engine. The hollow blade rotor is functioning as radial compressor, heat is generated in the two dimensional combustion chamber, and the rotor blades work as a turbine. The proposed test rig is shown in the Fig. 3.

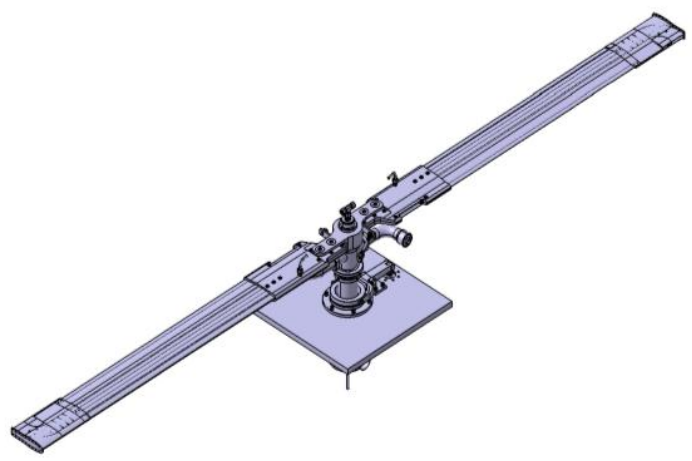

Fig. 3. Rotor test rig. 


\subsection{Tip-jet combustion chamber}

In order to decrease aerodynamic drag, 2-dimensional combustion chamber combustion chamber has been proposed. And for stability, pilot-can type ramjet combustor design logic is considered. The dimensional combustion chamber model is shown in Fig. 4. This combustion chamber is produced and tested in laboratory before adjusted to the rotor, by taking a special care to simulate outer wall cooling same as it would be at the rotor tip. The 2-dimensional combustion chamber model at test stand is shown in Fig. 5 .
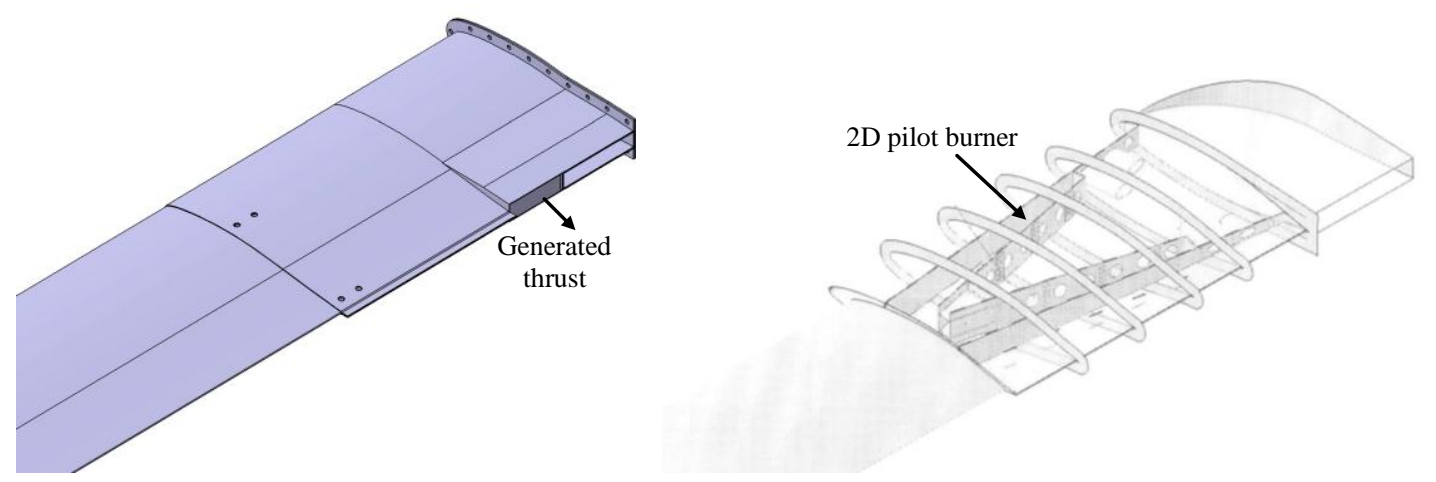

Fig. 4. 2-D combustion chamber.

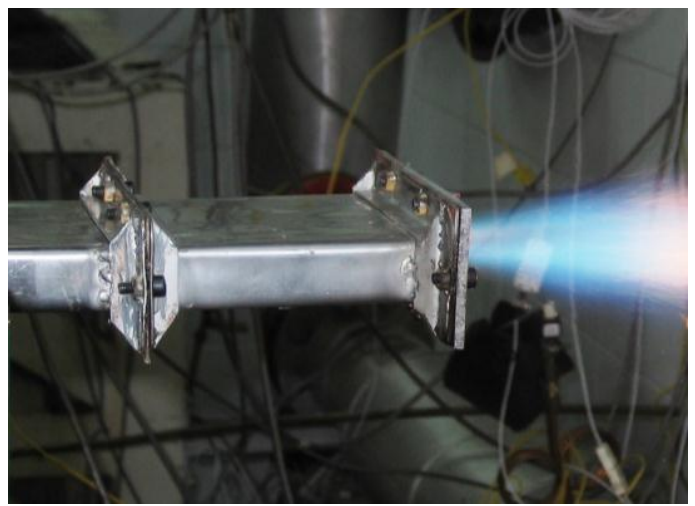

Fig. 5. 2-D combustion chamber model at test stand.

\subsection{Present model}

In the model considered in this work, the thrust produced at the rotor tip, is given by

$$
F=m_{a} V_{e}
$$

Power generated due to rotor tip jet is:

$$
\begin{aligned}
& P=n F V_{t}-P_{\text {drag }}=n m_{a} V_{e} V_{t}-n m_{a} V_{t}^{2} \\
& P=n m_{a}\left(V_{e}-V_{t}\right) V_{t}
\end{aligned}
$$

The pressure in the combustion chamber is determined fromthe pressure rising in the air compressor due to rotor centrifugal action in the blades, the pressure loss due to friction in the blade, and the pressure loss in the combustion chamber. Thus by considering the compression obtained in the rotor channel due to centrifugal force, the rotor useful power is: 


$$
P_{R O T}=n \cdot m_{a} \cdot\left\{\left[V_{e} \cdot V_{t}\right]-\left[V_{t}\right]^{2}-\left[W_{b}\right]\right\}
$$

where $W_{b}$ is the work used for compression through rotor blades:

$$
W_{b}=c_{p a}(T) \cdot T_{a} \cdot\left(\frac{\pi_{r}^{\frac{\gamma-1}{\gamma}}-1}{\eta_{r}}\right)
$$

and thus:

$$
P_{R O T}=n m_{a}\left\{\left[V_{e} V_{t}\right]-\left[V_{t}\right]^{2}-\left[c_{p a}(T) T_{a}\left(\frac{\pi_{r}^{\frac{\gamma-1}{\gamma}}-1}{\eta_{r}}\right)\right]\right\}
$$

Specific usefull power

$$
P_{s p}=\frac{P_{R O T}}{m_{a}}
$$

Specific consumption

$$
C_{s p}=\frac{m_{f}}{P_{R O T}}
$$

And thus crusing time is

$$
\tau(M)=-\int_{M}^{M_{v}} \frac{d m}{C_{s p} P_{R o T}(m)}
$$

The pressure in the combustion chamber is determined from the pressure rise in the air compressor due to rotor centrifugal action in the blades, the pressure loss due to friction in the blade, and the pressure loss in the combustion chamber

\section{Results and Comparison}

For the present model, analysis was performed for helicopter rotor with two blades, airfoil NACA 0018, chord length $250 \mathrm{~mm}$ and rotor radius $2.75 \mathrm{~m}$. Burner exit temperature was $1900 \mathrm{~K}$, burner efficiency and burner pressure drop were calculated using data from combustion chamber tests.

In comparing the payload endurance characteristics for the different types of helicopter power systems, the optimum condition for all types is considered. A value of $v_{\text {tip }}=280 \mathrm{~m} / \mathrm{s}$ is taken, and it forms a reasonable basis for comparison for pressure jet system.

The comparisons curves of payload endurance for configurations of $300 \mathrm{~kg}$, and $100 \mathrm{~kg}$ are given in Fig. 6. This graph shows the general implications of present pressure jet power system and rocket power system. The rocket jet rotor helicopter can carry a considerably greater payload, but as shown in the Fig. 7 it can only operate for very short time that is because the engine has higher fuel consumption than a pressure combustion engine. Thus pressure combustion power system is much better than rocket power system; because of its lower specific consumption.

And required power for the suggested tip jet rotor helicopter is given against tip speed in Fig. 8, with maximum mass of $350 \mathrm{~kg}$, mass of fuel $200 \mathrm{~kg}$. Air mass flow rate against tip speed is shown in Fig. 9. 


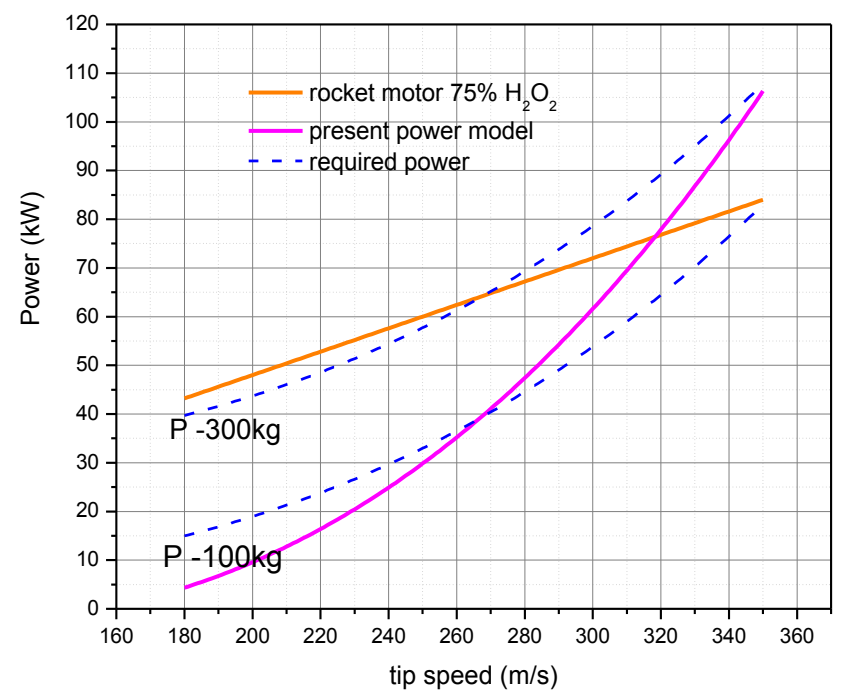

Fig. 6. Payload endurance for configurations of $300 \mathrm{~kg}$, and $100 \mathrm{~kg}$.

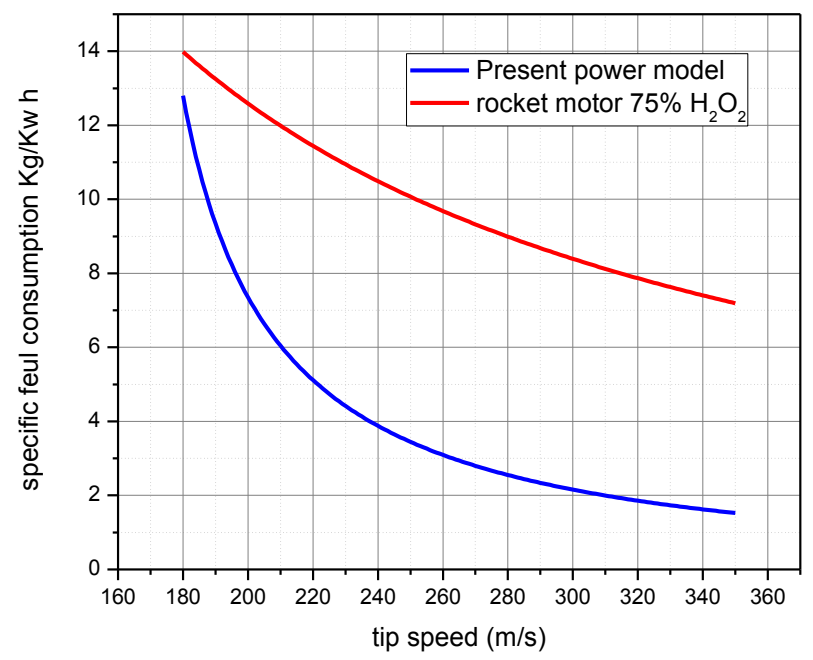

Fig. 7. Systems fuel consumption.

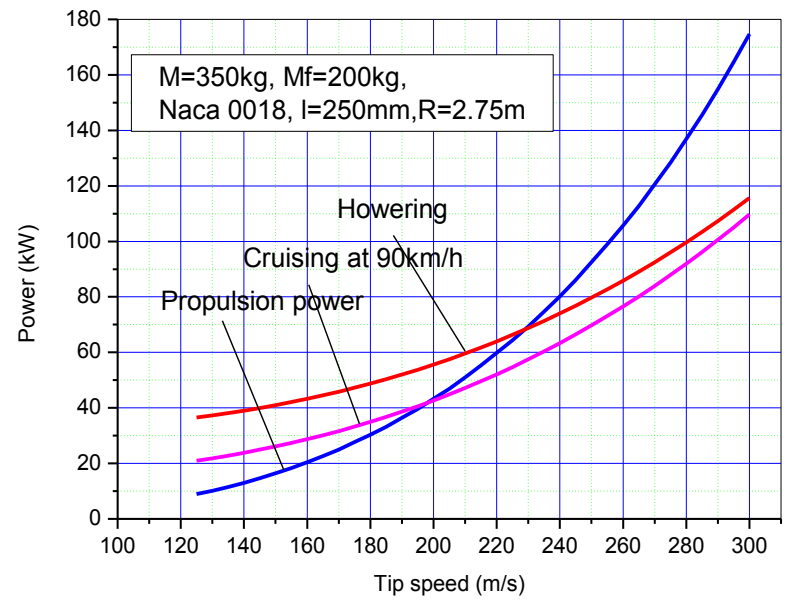

Fig. 8. Required and delivered power vs. tip speed. 


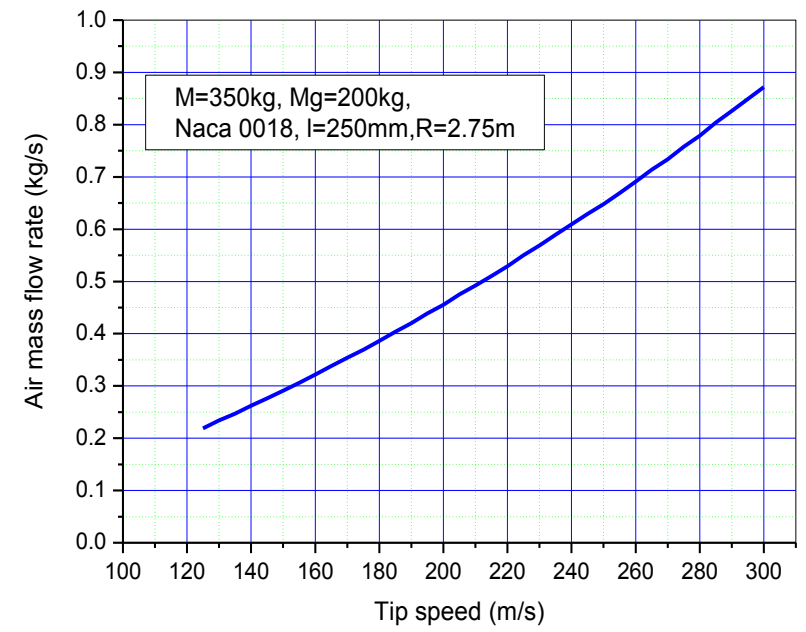

Fig. 9. Air mass flow rate through single blade vs. tip speed.

\section{Results and Comparison}

It has been shown that, for a helicopter of a given gross weight, the rocket jet rotor configuration is capable of a considerably higher payload for flight of very short time, but that only for short flights are possible. What characterizes a rocket engine is that it can develop a high power despite being light weighted, and the drawback is that the engine has higher fuel consumption than a pressure combustion engine. So the rocket engine is best suited for applications where low weight is important and high power is needed for a shorter period of time. Thus, the rocket jet rotor system helicopter will therefore have only limited specialized application. The present pressure combustion power system operates much better than the rocket system if saving in fuel consumption is considered with the simplicity of the unit design.

For the present model, the required tip speed is at least $200 \mathrm{~m} / \mathrm{s}$, appropriate mass flow rate is about 0.5 $\mathrm{kg} / \mathrm{s}$. It means that required dimensions for combustion chamber would cause difficulties in order to satisfy low drag and mass from one side and low pressure drop and good efficiency from another side. Tests which were performed with combustion chamber showed that appropriate 2D-design can meet these challenges. Flight time of such configuration is of the order of one hour, cruising at $90 \mathrm{~km} / \mathrm{h}$. Chosen configuration can meet demands for expendable vehicle used for unmanned exploration, rescue and special military missions

\section{References}

[1] Stewart W., Burle M.F., The application of jet propulsion to helicopters, A.R.C. Technical Report, London 1950.

[2] Henry J.R., One dimensional, compressible, viscous flow relations applicable to flow in a ducted helicopter blade, NACA TN3089, Washington 1953.

[3] Krebs R.P. and Miller W.S., Analysis of pressure-jet power plant for a helicopter, NACA RM-E54L23, Washington 1955.

[4] Mavris D.N., Tai J.C., and Schrage D.P., A multidisciplinary design optimization approach to sizing stopped rotor configurations utilizing reaction drive and circulation control. Paper presented at the 5th AIAA/NASA/USAF/ISSMO Symposium on Multi-disciplinary Analysis and Optimization, Panama City, Florida, September 7-9, 1994.

[5] Liberatore E.K., Cold cycle pressure jet helicopters ventures designs and developments, VERTIFLITE January/February 1992, $38(1)$.

[6] Aiman E. and Algattus S. S., Analysis of different propulsion systems for tip jet rotor system, Journal of Engineering Research, Faculty of Engineering Al-fateh University, 16th year2011.

[7] Kyung-Hoon P., Nam S. G., Hoon C. P., Kwang J. Y., and Yung H., Byun Design and test of power system for a reduced scale tip jet rotor using a small turbojet engine. AIAA September 2003-6570

[8] George P. Sutton, Rocket Propulsion Elements, Seventh Edition, by John Wiley \& Sons, Inc., 2001. 